\title{
Percentual de Contas recebidas e devolvidas do Faturamento para o Setor de Auditoria de Contas Médicas de em um Hospital Particular do Recife
}

\author{
Grace Mary Gomes Cintra ${ }^{1}$; Carla Giselly de Souza ${ }^{2}$; Marta Gerusa Silva de Souza ${ }^{3}$; Gislaine Farias de Oliveira ${ }^{4}$
}

\begin{abstract}
Resumo: A atenção à saúde não é gratuita e sejam quais forem as fontes de financiamento, públicas ou privadas, elas absorvem recursos na forma de impostos, seguros ou despesas particulares. O sistema brasileiro de saúde tem passado por uma crise financeira, sem precedentes, crise esta resultante da má administração financeira destes recursos. A palavra Auditoria vem do latim Auditore, que significa aquele que ouve, ouvidor. É o perito encarregado de examinar contas. O processo de auditoria consiste em analisar documentos, registros, orçamentos, apresentações de fatos ou outras informações e depois comparar com uma norma ou requisito préestabelecido. A glosa é o cancelamento parcial ou total destes itens, por serem considerados ilegais ou indevidos, ou seja, quando o auditor do plano de saúde não considera cabível o pagamento. Faturamento é o conjunto dos recebimentos expressos em unidades monetárias, obtidas por uma empresa com a venda de bens ou serviços em determinado período, em outras palavras é o número de unidades de bens ou serviços vendidos multiplicado pelo preço de venda unitário. Foi possível observar que o convenio que mais atendeu durante o ano de estudo foi o Sulamérica, com um total de $17,67 \%$ dos atendimentos realizados no ano, seguido do convênio Unimed com $14,75 \%$ e Saúde Bradesco 13,72\%. Para que possamos diminuir o percentual de perdas hospitalares é necessário um planejamento prático e eficaz, objetivando a pré-análise, que deve ser feita por profissionais de saúde qualificados possuidores de uma visão crítica e propositora de sua atuação, onde, devem desempenhar suas atividades focando sempre na qualidade da assistência prestada aos pacientes desde sua admissão até a alta hospitalar, acompanhando todos os procedimentos realizados, comparando prescrição com a saída de material médico da farmácia, monitorando solicitações de exames, lançando taxas, observando descrição e a evolução médica e de enfermagem, orientar os profissionais, quanto a importância de uma evolução fiel, real técnica.
\end{abstract}

Palavras- chave: Glosas, Conveniados, Faturamento hospitalar.

\section{Received and Returned Bills percentage to the Billing Sector Audit of Medical Bills in a Recife's Private Hospital}

\begin{abstract}
Health care attention is not free, whatever the sources of funding, public or private, they absorb resources in the form of taxes, insurance or personal expenses. The Brazilian health system has gone through a financial crisis, unprecedented crisis is a result of financial mismanagement of these resources. The word Audit comes from Latin Auditore, which means one who listens, ombudsman. He is the expert in charge of examining bills. The audit process is to analyze documents, records, budgets, presentation of facts or other information and then compare to a standard or requirement preset. The gloss is the partial or total removal of these items because they are considered illegal or improper, in other words, when the auditor's health plan does not consider appropriate payment. Billing is all the receipts expressed in monetary units, obtained by a company from selling goods or services in a certain period, in other words is the number of units of goods or services sold multiplied by the unit selling price. It was observed that the covenant that most attended during the study was Sulamérica, with a total of $17.67 \%$ of visits made in the year, followed by agreement with Unimed $14.75 \%$ and $13.72 \%$ Bradesco Health. So we can reduce the percentage of losses hospital planning is necessary practical and effective, aimed at pre-analysis, which must be made by qualified health professionals who hold a critical view
\end{abstract}

\footnotetext{
${ }^{1}$ Enfermeira, Especialista em Educação em Saúde UFPE- 2003 e Especialista em Auditoria de Saúde CEFAPP 2011. Endereço para correspondência: Rua Dos Navegantes, 1907/501, Boa Viajem, Recife, Pernambuco. Contato: gracecintra@hotmail.com

${ }^{2}$ Orientadora, Zootecnista, Doutora em Nutrição Animal. Endereço para correspondência: Rua Central, 50, Massangana, Jaboatão dos Guararapes-PE. Contato:. carlaxlsouza@yahoo.com.br.

${ }^{3}$ Enfermeira, Especialista em Auditoria de Saúde- CEFAPP 2011. Endereço para correspondência: Rua Central, 50,

Massangana, Jaboatão dos Guararapes, Pernambuco. Contato: martadeu@ oi.com.br

${ }^{4}$ Administradora de Empresas pela Faculdade Olindense de Administração e Ciências Contábeis. Acadêmica de Ciências Contábeis pela Faculdade Metropolitana - PE. Contato: gislainefarias44@gmail.com.
} 
of its performance and willful, where shall perform its activities focusing always the quality of care provided to patients from admission to discharge, following all the procedures performed, comparing prescriptions with the output of medical pharmacy, monitoring requests for tests, launching fees, noting description and evolution, medical and nursing, guide professionals, and the importance of a faithful development, real technical.

Keywords: Glosses, agreements, hospital billing.

\section{Introdução}

A atenção à saúde não é gratuita e sejam quais forem as fontes de financiamento, públicas ou privadas, elas absorvem recursos na forma de impostos, seguros ou despesas particulares, sendo que esses recursos não são ilimitados (CILE, 1993). As necessidades de saúde são ilimitadas enquanto os recursos econômicos disponíveis para atendê-las são escassos (ANSELMI e NAKAO, 1999).

O sistema brasileiro de saúde tem passado por uma crise financeira, sem precedentes, crise esta resultante da má administração de recursos sejam eles públicos ou privados. Assim, custos crescentes e elevados dos serviços de saúde têm afetado todos os prestadores de serviço (COUTTOLENC e ZUCCHI, 1998). Deste modo, a auditoria em Serviços de Saúde é um tema dos mais relevantes às Instituições de Saúde, porquanto essencial à manutenção da própria "saúde financeira" de tais organizações (MAIA e PAES, 2005).

A palavra Auditoria vem do latim Auditore, que significa aquele que ouve, ouvidor. É o perito encarregado de examinar contas. Atividade de avaliação independente e de assessoramento do escalão superior na administração, voltada para o exame e análise da adequação, eficiência (a ação), eficácia (o resultado), efetividade (o desejo; custo/benefício), e qualidade nas ações de saúde, praticadas pelos prestadores de serviço, sob os aspectos quantitativos (produção e produtividade), qualitativos e contábeis (custos operacionais), com observância de preceitos éticos e legais (MAIA e PAES, 2005).

O processo de auditoria consiste em analisar documentos, registros, orçamentos, apresentações de fatos ou outras informações e depois comparar com uma norma ou requisito pré-estabelecido. 
A glosa é o cancelamento parcial ou total destes itens, por serem considerados ilegais ou indevidos, ou seja, quando o auditor do plano de saúde não considera cabível o pagamento. As glosas são aplicadas quando houver qualquer situação que gera dúvidas em relação a regra e prática adotada pela instituição de saúde (FRANCISCO, 1993).

A auditoria de enfermagem incorporou-se à rotina das instituições de saúde com o intuito de avaliar os aspectos qualitativos da assistência requerida pelo paciente, os processos internos e as contas hospitalares (SCARPARO et al., 2009).

A auditoria de enfermagem representa a função de controle do processo técnico e administrativo, verificando se os resultados da assistência estão de acordo com os objetivos (SILVA et al., 2009). Dessa forma, vem sendo concebida como o exame oficial dos registros de enfermagem com o objetivo de avaliar, verificar e melhorar a assistência, podendo concentrar-se nos registros e anotações de enfermagem (LOPES, 1998).

A auditoria tem surgido como uma ferramenta importante para mensuração da qualidade (Auditoria de cuidados) e custos (Auditoria de custos) das instituições de saúde. O processo de auditoria é conceituado como uma avaliação sistemática e formal de uma atividade realizada por pessoas não envolvidas diretamente em sua execução a fim de se determinar se a atividade está de acordo com os objetivos propostos (GOTO 2001). Desta forma, é possível evidenciar deficiências nas atividades desenvolvidas e apontar alternativas preventivas e corretivas para as mesmas (FARACO E ALBUQUERQUE, 2004).

A NBR ISO 19011 (2002) define auditoria com um "processo sistemático, documentado e independente para obter evidências de auditoria (registros, apresentação de fatos ou outras informações) e avaliá-las objetivamente para determinar a extensão na qual os critérios da auditoria (conjunto de políticas, procedimentos ou requisitos) são atendidos".

A portaria 169 do Ministério da Saúde (MS), do gabinete do ministro, de 19 de agosto de 1999, estabelece uma nova organização de atividades do SNA: as de controle e avaliação ficam com a Secretaria de Assistência a Sade (SAS), as de auditoria, com o Departamento Nacional de Auditoria do SUS (DENASUS), representada em todos os estados da federação no distrito federal. Em dezembro de 1999, foi criada a Sociedade Brasileira em Enfermeiros Auditores em Saúde (SOBEAS), tendo como finalidade agregar profissionais de todo país, fossem enfermeiros envolvidos ou interessados em auditoria (BRASIL, 2007; SILVA et al., 2009). 
A Normatização do COREN (2001) diz que é da competência privativa do Enfermeiro Auditor no exercício de suas atividades: organizar, dirigir, planejar, coordenar e avaliar, prestar consultoria, auditoria e emissão de parecer sobre os serviços de Auditoria de Enfermagem.

A Auditoria Médica, antes de ser uma necessidade, é uma questão de qualidade comprometida com a verdade. Sendo assim, o processo de auditoria respeita sempre os mesmos princípios técnicos e éticos, independentemente da origem do usuário ${ }^{4}$. Neste aspecto, a atuação da Auditoria em contas médicas está voltada para verificação de códigos solicitados, autorizados ou não, corrigindo eventuais distorções, evitando cobrança incorreta e a conseqüente glosa.

Maia e Paes, (2005) relata que o Auditor tem como atribuição subsidiar os setores de análise com informações relevantes para o correto pagamento das contas e ser um elemento de ligação entre os usuários e a empresa patrocinadora do evento agilizando a parte técnica e dando suporte administrativo.

O papel da enfermagem na auditoria é avaliar a assistência que o paciente está recebendo, assim como a integralidade e exatidão da documentação dessa assistência no prontuário. Limita-se à avaliação dos cuidados de enfermagem prestados ao paciente, daí a importância de uma ação integrada com o auditor médico, para se ter uma visão da assistência global prestada ao paciente (MAIA e PAES, 2005).

Deste modo esta pesquisa objetivou avaliar o percentual total de contas médicas faturadas no ano de 2015, em um Hospital particular do Recife, observando, quantidades e valores enviados aos prestadores de saúde suplementar, após auditoria e correções para pagamento.

\section{Metodologia}

Trata-se de uma pesquisa de abordagem quantitativa, descritiva e exploratória no setor de contas médicas de um grande Hospital particular de Recife. 
A pesquisa foi realizada no setor de Contas médicas, onde avaliou-se a quantidade de contas (fatura/ prontuário) enviadas e devolvidas pelo faturamento ao setor de contas médicas no referido hospital durante o ano de 2015. O critério de pesquisa pautou-se pelos meses do ano, número de contas e valores, recebidas e devolvidas, ao faturamento do hospital para cobrança ao convenio. A coleta de dados foi feita através dos relatórios de produção anual com base no relatório de diligenciamento sintético emitido pelo setor de contas médicas das 07:00 horas do dia 01/01/2015 até as 23:59 do dia 31/12/2015.

\section{Resultados e Discussão}

$\mathrm{Na}$ tentativa de identificar o valor das contas auditadas enviadas e devolvidas pelo faturamento do Hospital particular, durante o ano de 2015 e evidenciar os principais convênios de saúde associados, apresentamos os resultados nas Tabelas 1, 2 e 3 .

Para evitar glosas, a maioria das instituições de saúde elabora seus fluxogramas com a pré-análise efetuada pelas enfermeiras.

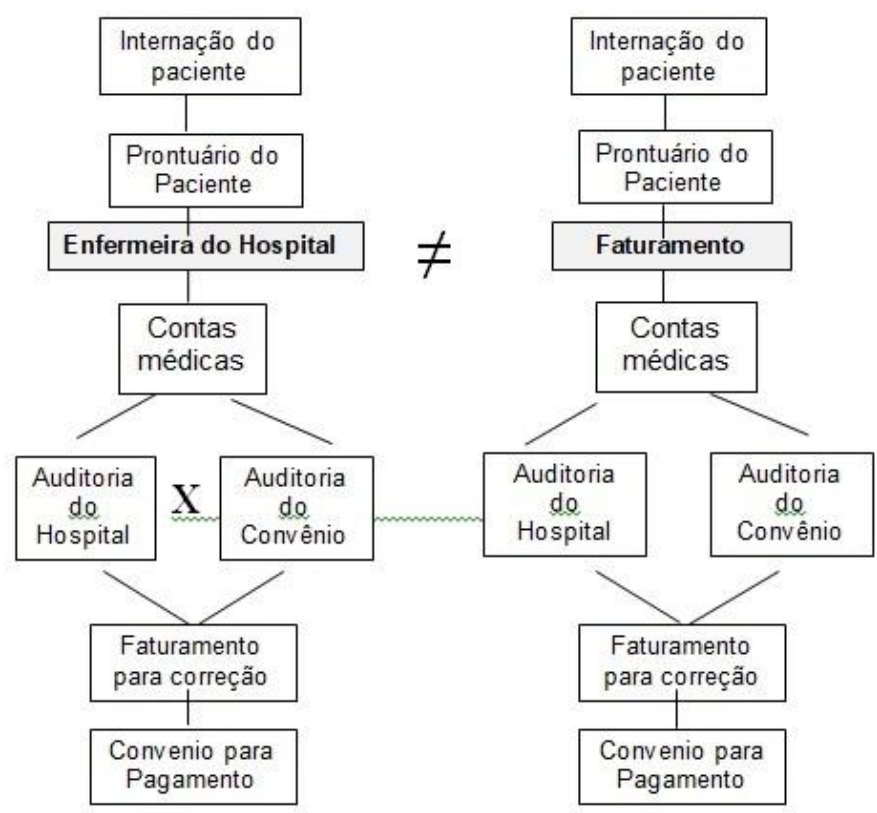

Figura 1- Fluxograma de Algumas instituições hospitalares
Figura 2- Fluxograma da instituição em estudo 
Porém no hospital abordado, o formato do fluxo das contas hospitalares a serem auditadas seguem um roteiro diferente, deste modo expomos abaixo, nas Figuras 1 e 2, um fluxograma explicativo /comparativo que justifica a maneira de atuação de cada instituição conforme a própria atuação:

Apesar de o hospital estudado utilizar o fluxograma 02 (dois), é importante ressaltar que o mais eficaz é o numero 01(um), utilizado pela maioria dos hospitais, porém, devido ao seu grande porte o hospital em estudo não conseguiu se amoldar à metodologia dos demais, onde, atualmente seria necessária a admissão de uma média de 30 (trinta) enfermeiros, a serem distribuídos nos postos de enfermagem e no setor de faturamento, que para os gestores seria um grande custo, porém, se compararmos as demais instituições podemos comprovar que a economia com o materiais utilizados e redução nas glosas, ultrapassaria este investimento. Salientamos ainda, a grande importância das pré análises realizadas pelas enfermeiras das instituições hospitalares.

A Tabela 1 a seguir, refere-se a contas recebidas do faturamento para serem analisadas pelos auditores externos (convênios), tendo como segundo tempo a análise e discussão das divergências.

Tabela 1- Relatório de produção mensal 2015 do Setor de Contas Médicas, com número de contas recebidas e devolvidas do faturamento

\begin{tabular}{c|c|c|c|c|c}
\hline Mês & $\begin{array}{c}\text { Contas } \\
\text { Recebidas }\end{array}$ & Valores & $\begin{array}{c}\text { Contas } \\
\text { Devolvidas }\end{array}$ & Valores & Diferença \\
\hline Janeiro & 1.566 & $\mathrm{R} \$ 15.933 .214,22$ & 1.416 & $\mathrm{R} \$ 13.644 .147,88$ & -150 \\
\hline Fevereiro & 1.234 & $\mathrm{R} \$ 13.120 .888,33$ & 1.128 & $\mathrm{R} \$ 11.047 .489,65$ & -106 \\
\hline Março & $\mathbf{1 . 7 1 3}$ & $\mathrm{R} \$ \mathbf{1 8 . 0 7 7 . 2 7 7 , 6 5}$ & $\mathbf{1 . 9 5 9}$ & $\mathbf{R} \mathbf{1 8 . 6 0 9 . 1 4 6 , 5 5}$ & $+\mathbf{2 4 6}$ \\
\hline Abril & 1.591 & $\mathrm{R} \$ 16.984 .875,66$ & 1.386 & $\mathrm{R} \$ 13.494 .873,81$ & -205 \\
\hline Maio & 1.812 & $\mathrm{R} \$ 19.037 .841,64$ & 1.465 & $\mathrm{R} \$ 15.966 .941,94$ & -347 \\
\hline Junho & 1.407 & $\mathrm{R} \$ 16.604 .643,24$ & 1.447 & $\mathrm{R} \$ 14.592 .539,64$ & -40 \\
\hline Julho & 1.766 & $\mathrm{R} \$ 20.157 .245,99$ & 1.657 & $\mathrm{R} \$ 15.630 .308,42$ & -109 \\
\hline Agosto & 2.064 & $\mathrm{R} \$ 21.309 .938,26$ & 1.915 & $\mathrm{R} \$ 18.544 .161,45$ & -149 \\
\hline Setembro & 1.882 & $\mathrm{R} \$ 20.380 .325,86$ & 1.758 & $\mathrm{R} \$ 19.089 .742,19$ & -124 \\
\hline Outubro & $\mathbf{1 . 7 1 8}$ & $\mathbf{R} \$ \mathbf{1 9 . 2 7 6 . 9 3 0 , 4 4}$ & $\mathbf{1 . 8 2 1}$ & $\mathbf{R} \mathbf{1 7 . 5 6 2 . 7 1 4 , 4 0}$ & $+\mathbf{1 0 3}$ \\
\hline Novembro & 1.694 & $\mathrm{R} \$ 20.204 .735,48$ & 1.672 & $\mathrm{R} \$ 17.955 .458,23$ & -20 \\
\hline Dezembro & 1.647 & $\mathrm{R} \$ 20.347 .778,91$ & 1.526 & $\mathrm{R} \$ 18.169 .091,60$ & -121 \\
\hline Total & $\mathbf{2 0 . 0 9 4}$ & $\mathbf{R} \$ \mathbf{2 2 1 . 4 3 5 . 9 6 5 , 6 8}$ & $\mathbf{1 9 . 1 5 0}$ & $\mathbf{R} \mathbf{1 9 4 . 3 0 6 . 6 4 2 , 7 6}$ & $\mathbf{1 . 0 2 2}$ \\
\hline
\end{tabular}


Observa-se que durante 10 meses, a quantidade de contas enviadas ao faturamento foi menor do que a recebida, isto significa retenção do número de contas e conseqüentemente dos valores que decorrem de vários fatores, como; falta de autorização de orteses, próteses e materiais especiais, falta de autorização de procedimento, falta de prorrogação de diária, entre outros.

Observa-se que o número de contas dos meses de março e outubro demonstram a diferença da quantidade está relacionada a retenções anteriores, por motivos já descritos. Porém no me de outubro apesar de ter sido enviado ao faturamento quantidade maior de contas, os valores não seguiram o mesmo padrão devido a contas complementares ou de menor valor e retenção das próprias contas de outubro.

Tabela 2 - Índice de contas hospitalares enviadas aos Auditores pelo setor de faturamento no ano de 2015 conforme convenio de saúde

\begin{tabular}{l|c|c|c}
\hline \multicolumn{1}{c|}{ Convênio } & Quantidade & \% & Valor da conta \\
\hline AGF & 1 & 0,005 & $1.539,71$ \\
\hline AMEPE & 67 & 0,33 & $684.652,21$ \\
\hline AMIL & 102 & 0,05 & $1.493 .990,74$ \\
\hline ASSEFAZ & 406 & 2,0 & $6.044 .746,62$ \\
\hline BACEN & 104 & 0,5 & $11.957 .394,9$ \\
\hline CAMED & 1262 & 7,6 & $13.689 .754,40$ \\
\hline CASSI & 1513 & 7,49 & $18.505 .179,15$ \\
\hline CONAB & 31 & 0,15 & $245.321,91$ \\
\hline CORREIOS & 773 & 3,85 & $8.794 .063,45$ \\
\hline EMBRATEL & 67 & 0,33 & $1.259 .412,72$ \\
\hline FACHESF & 1116 & 5,72 & $14.120 .029,79$ \\
\hline FASSINCRA & 143 & 0,71 & $2.176 .851,21$ \\
\hline FIOPREV & 66 & 0,32 & $962.872,01$ \\
\hline FUN MEDIAL & 1072 & 5,34 & $5.941 .681,1$ \\
\hline FUNCEF SAÚDE CAIXA & 352 & 1,74 & $4.291 .198,17$ \\
\hline FUSEX & 296 & 1,46 & $5.110 .428,20$ \\
\hline GAMA SAUDE LTDA & 1 & 0,01 & $17.763,62$ \\
\hline GEAP & 218 & 1,08 & $2.160 .409,86$ \\
\hline GRUPO SERV. MED & 9 & 0,04 & $147.637,33$ \\
\hline IDEAL SAÚDE & 25 & 0,12 & $356.310,1$ \\
\hline INFRAERO & 4 & 0,02 & $37.960,06$ \\
\hline MEDIAL SAÚDE & 507 & 1,51 & $3.273 .419,4$ \\
\hline MEDISERVICE & 316 & 1,56 & $3.125 .529,20$ \\
\hline MINIST. PUBLICO & 5 & 0,024 & $37,192,94$ \\
\hline PETROBRAS PETROL. & 173 & 0,85 & $1.804 .863,2$ \\
\hline
\end{tabular}


Id on Line Revista Multidisciplinar e de Psicoloqia

Id on Line Multidisciplinary and Psycology Journal

\begin{tabular}{l|c|c|c}
\hline PROASA APTO & 2 & 0,01 & $2.945,38$ \\
\hline S. BRADESCO & $\mathbf{2 7 4 4}$ & $\mathbf{1 3 , 7 2}$ & $\mathbf{3 0 . 5 3 4 . 6 7 0 , 1}$ \\
\hline S. EXCELCIOR & 2230 & 11,03 & $10.059 .858,3$ \\
\hline SANTA CLARA & 23 & 0,11 & $4.774,00$ \\
\hline SECRET. SAÚDE IRH & 3 & 0,01 & $51.224,28$ \\
\hline SULAMERICA & $\mathbf{3 5 8 2}$ & $\mathbf{1 7 , 6 7}$ & $\mathbf{4 6 . 0 1 8 . 5 0 2 , 3}$ \\
\hline UNAFISCOSAUDE & 24 & 0,12 & $528.931,71$ \\
\hline UNIBANCO AIG SAUDE & 5 & 0,02 & $19.727,35$ \\
\hline UNIMED & $\mathbf{2 9 8 2}$ & $\mathbf{1 4 , 7 5}$ & $\mathbf{2 6 . 1 8 2 . 7 1 7 , 0 8}$ \\
\hline \multicolumn{1}{c|}{ Total } & $\mathbf{2 0 2 0 4}$ & $\mathbf{1 0 0}$ & $\mathbf{2 0 9 . 0 7 6 . 6 0 5 , 8 9}$ \\
\hline
\end{tabular}

A Tabela 2 demonstra o valor gerado nominalmente por cada convenio no ano de 2015 que foram analisados pela auditoria do Hospital X Convenio, e enviados para faturamento para serem corrigidos. O convenio que mais atendeu durante $\mathrm{o}$ ano de estudo foi $\mathrm{o}$ Sulamérica, com um total de $17,67 \%$ dos atendimentos realizados no ano, seguido do convênio Unimed com 14,75\% e Saúde Bradesco 13,72\%. Zunta, (2009) durante dois meses de 2005 avaliou em um grande hospital no Estado de São Paulo o faturamento da UTI, onde constatou que existiu uma dominância de $27,7 \%$ de um determinado plano de saúde e de apenas $13,2 \%$ para o segundo colocado, fator que, de certo modo concorda com o encontrado neste estudo, demonstrado que a utilização de planos de saúde é bem heterogênea, com o aparecimento de vários convênios de saúde, porém com poucos demonstrando grande dominância de mercado.

Embora a maioria dos preços pagos pelos convênios de saúde sejam iguais, pode haver uma diferenciação no faturamento dos itens relacionados ao tipo de convenio, portanto, o mesmo procedimento, material ou medicamento podem ter faturamentos diferentes, em função de acordos feitos entre os convênios e o hospital, bem como preços que são atualizados e outros convênios mantém os anteriores (ZUNTA, 2009).

A questão de custos na área de saúde tem exigido profissionais cada vez mais qualificados que cooperem no resultado econômico da instituição. Dado que as perdas de materiais e medicamentos- as principais fontes lucrativas do hospital- são pouco controladas, a auditoria em enfermagem pode desempenhar um trabalho proativo em semelhança a este aspecto (D'INOCENZO, 2006). 
Tabela 3- Índice de contas hospitalares analisadas devolvidas do faturamento no ano de 2015 conforme convenio de saúde

\begin{tabular}{|c|c|c|}
\hline Convênio & Quantidade & Valor da conta \\
\hline AMEPE & 70 & $683.455,95$ \\
\hline AMIL & 86 & $1.372 .422,99$ \\
\hline ASSEFAZ & 386 & $5.760 .898,28$ \\
\hline BACEN & 92 & $1.044 .271,07$ \\
\hline CAMED & 1270 & $19.450 .189,77$ \\
\hline CASSI & 1341 & $15.823 .431,99$ \\
\hline CONAB & 34 & $252.045,23$ \\
\hline CORREIOS & 722 & 7.012 .264 .53 \\
\hline EMBRATEL & 65 & $1.284 .097,56$ \\
\hline FACHESF & 1099 & $13.734 .213,36$ \\
\hline FASSINCRA & 136 & $2.058 .146,02$ \\
\hline FIOPREV & 43 & $520.041,24$ \\
\hline FUN MEDIAL & 948 & $4.626 .218,26$ \\
\hline FUNCEF SAÚDE CAIXA & 329 & $9.963 .898,70$ \\
\hline FUSEX & 290 & $4.444 .650,32$ \\
\hline GEAP & 197 & $1.868 .772,75$ \\
\hline GRUPO SERV. MED & 14 & $244,654.99$ \\
\hline IDEAL SAÚDE & 28 & $583.624,55$ \\
\hline MEDIAL SAÚDE & 466 & $2.532 .821,68$ \\
\hline MEDISERVICE & 295 & $2.939 .007,48$ \\
\hline PETROBRAS PETROL. & 163 & $1.664 .181,10$ \\
\hline S. BRADESCO & 2640 & 30.212.191,11 \\
\hline SAÚDE EXCELCIOR & 2099 & $8.798 .065,61$ \\
\hline SULAMERICA & 3292 & 41.284.716,65 \\
\hline UNAFISCOSAUDE & 25 & $535.041,01$ \\
\hline UNIMED & 2805 & 24.348.652,99 \\
\hline Total & 18935 & 191.042.347,19 \\
\hline
\end{tabular}

A Tabela 3 demonstra o valor gerado nominalmente por cada convenio no ano de 2015 das contas que foram analisados pela auditoria do Hospital X Convenio, e devolvidas do faturamento já corrigidas. Mesmo com as correções efetuadas de contas glosadas, ainda assim o convenio que mais atendeu durante o ano de estudo foi o Sulamérica, seguido do convênio Unimed e Saúde Bradesco. 


\section{Considerações Finais}

Observou-se no presente estudo que a diferença percentual de economia gerada entre as contas recebidas e devolvidas foi de $8,62 \%$.

Para que possamos diminuir o percentual total de perdas hospitalares é necessário um planejamento prático e eficaz, tendo seu maior objetivo a pré-análise, esta deve ser feita por profissionais de saúde qualificados que tendo uma visão crítica e propositora de sua atuação, onde, devem desempenhar suas atividades focando sempre na qualidade da assistência prestada aos pacientes desde sua admissão até a alta hospitalar, acompanhando todos os procedimentos realizados, comparando prescrição com a saída de material médico da farmácia, monitorando solicitações de exames, lançando taxas, observando descrição, evoluções médicas e de enfermagem, orientar os profissionais, quanto a importância de uma evolução fiel, real técnica. Ser um constante educador.

É necessário também que o faturamento hospitalar seja realizado por técnicos especializados, de preferência com experiência assistencial, porque faturar não é contar simplesmente, é viver cada situação como se fosse a tempo real, para oferecer ao seu paciente um tratamento eficaz e econômico.

A atual realidade vivida pelos profissionais de enfermagem em seus locais de trabalho, tem exigido dos mesmos conhecimentos, habilidades e atitudes na área de gestão de custos. Portanto, sugere-se a enfermeira que, deseja ter um diferencial em sua profissão, não só levantar custos de sua assistência, mas também conhecer a sua contribuição no faturamento da organização.

\section{Referências}

ANSELMI, M.A.; NAKAO, J.R.S. A Enfermagem no processo de gestão economica dos serviços de saúde: Limites e possibilidades. Rev. Bras. de Enfermagem. 1999, 52(2): 223-32.

BRASIL, Ministério Da Saúde: História da auditoria em saúde. [online]. Disponível em: www.sna.saude.gov.br/his-toria.cfm, 2007. 
COUTTOLENC, B.F.; ZUCCHI, P. Gestão de recursos financeiros. São Paulo, Faculdade de Saúde Pública da USP; 1998 (Série saúde e cidadania,10).

Consejo Internacional De Las Enfermerías. La calidad, los costos y la enfermería. [Presentado en el día internacional de la enfermería; Geneva; 1993].

D`INNOCENZO, M.; et al. Indicadores, Auditorias, Certificações: Ferramentas de Qualidade para Gestão em Saúde. São Paulo (SP): Martinar, 2006.

FARACO, M.M.; ALBUQUERQUE, G.L. Auditoria do método de assistência de enfermagem. Rev. Bras. Enferm. 2004; 57(4): 421-4.

FRANCISCO, M.T.R. Auditoria em enfermagem: padrões, critérios de avaliação e instrumentos. $3^{\text {a }}$ ed. São Paulo: Cedas; 1993.

GOTO, D.Y.N. Instrumento de auditoria técnica de conta hospitalar mensurando perdas e avaliando a qualidade da assistência [monografia]. Curitiba: Universidade Federal do Paraná; 2001.

International Organization For Standardization. NBR ISO 19011: Diretrizes para Auditorias de Sistema de Gestão da Qualidade e/ou Ambiental. ABNT, Rio de Janeiro, 2002

LOPES, C.M. Auditoria e distorções: ênfase nas atividades de anotações de enfermagem. Rev. Bras. Enferm. 1998; 51(1): 105-22.

MAIA, J.R.; PAES, P.P.L. Manual de Auditoria de contas Médicas. Hospital Geral de Juiz de Fora. 2005. Ministério da Defesa, Exército Brasileiro $4^{\mathrm{a}}$ Região Militar $/ 4^{\mathrm{a}}$ Divisão de Exército. Juiz de Fora, MG.

RESOLUÇÃO COFEN-266/2001, www.portalcofen.gov.br/node/4303

SCARPARO, A.F.; FERRAZ, C.A.; CHAVES, L.D.P.; ROTTA, C.S.G. Abordagem conceitual de métodos e finalidade da auditoria de enfermagem. Rev. Rene, Fortaleza, 2009. v. 10, n. 1, p. 1-165, jan./mar.

SILVA, S.H.; ORTIZ, D.C.F.; SHIMIZU, H.E.; TOTH, M. Auditoria em enfermagem: implantação e desenvolvimento no Hospital Universitário da Universidade de São Paulo. Rev. Esc. Enferm. USP 1990; 24(2): 1999-2009.

TORRES, L. Auditoria em enfermagem. [Tese de doutorado]. São Paulo: USP; 1977.

ZUNTA, R.S.B. O faturamento gerado pelos procedimentos de enfermagem em uma unidade de terapia intensiva. [Dissertação] São Paulo, 2009, USP, Escola de Enfermagem.11p.

Como citar este artigo (Formato ABNT):

GALDINO, Alzeneide dos S.; MARTINS, Jucinei dos S.; CELESTINO, Jurandi J.; RUFINO, Wasington S.; RODRIGUES, Luzia C. Reflexões sobre Gestão Escolar à partir da Percepção de Gestores da Rede Municipal. Id on Line Revista Multidisciplinar e de Psicologia, 2017, vol.11, n.37, p. 731-741. ISSN: 1981-1179.

Recebido: $25 / 09 / 2017$

Aceito: 29/09/2017 